\title{
An automated procedure for detection and identification of ball bearing damage using multivariate statistics and pattern recognition
}

\author{
Irina Trendafilova \\ Department of Mechanical Engineering, the University of Strathclyde \\ 75 Montrose street Glasgow, G1 1 XJ \\ Irina.Trendafilova@strath.ac.uk
}

phone: $+44(0) 141 / 5482322$

fax: $\quad+44(0) 141 / 5525105$

\begin{abstract}
.
This paper suggests an automated approach for fault detection and classification in roller bearings, which is based on pattern recognition and principal components analysis of the measured vibration signals. The signals recorded are pre-processed applying a wavelet transform in order to extract the appropriate high frequency (detailed) area needed for ball bearing fault detection. This is followed by a pattern recognition (PR) procedure used to recognise between signals coming from healthy bearings and those generated from different bearing faults. Four categories of signals are considered, namely no fault signals (from a healthy bearing) inner race fault, outer race fault and rolling element fault signals. The PR procedure uses the first six principal components extracted from the signals after a proper principal component analysis (PCA). In this work a modified PCA is suggested which is much more appropriate for categorical data. The combination of the modified PCA and the PR method ensures that the fault is automatically detected and classified to one of the considered fault categories. The method suggested does not require the knowledge/
\end{abstract}


determination of the specific fault frequencies and/or any expert analysis: once the signal filtering is done and the PC's are found the PR method automatically gives the answer if there is a fault present and its type.

Keywords: fault detection, vibration-based monitoring, ball bearing fault detection, signal analysis, pattern recognition, principal component analysis 


\section{\&1. Introduction}

Rolling element bearings constitute a major part of almost every rotating machine. There are a number of mechanisms that can lead to bearing damage and eventually failure, including mechanical damage, crack damage, wear damage, lubricant deficiency, corrosion and plastic deformation. When the smooth rolling contact surfaces are marred, higher stress conditions imposed on the surface reduce bearing life significantly. Thus the ability to detect bearing faults at an early stage is a major concern. Fault detection and identification in rolling element bearings has been a subject of extensive research over the past two decades [1-9,11-16]. Vibration-based monitoring is the most widely applied technique.

A fault in a ball bearing would normally create pulses with very low energy and wide-band spectra. On most occasions, especially when the fault is small, these pulses will be small and they will be buried in noise and in much higher energy low frequency components of the measured acceleration signal. This is the reason why ball bearing faults are not easy to detect. Since detecting and identifying ball bearing faults is quite a difficult job a lot of the methods suggested concentrate on making the detection possible and on the enhancement of the detection process rather than on making it automatic. Only a small number of papers address the importance of making the process automatic and attempt to develop an automatic procedure for ball bearing fault detection and/or identification. The advantage of an automatic procedure is that it does not require any further analysis and excludes the need for an experienced decision of whether or not there is a fault and/or what kind of fault it is to once the suggested transforms are done. This paper suggests a novel and easy to apply automatic process for ball bearing fault detection and identification, which does not 
require any human intervention. Thus the novelty and the contribution of this research is mainly in the development of an automatic process, which is capable of detecting and recognizing a ball bearing fault with a rather high success rate.

The application of traditional spectral analysis for ball bearing fault detection is somewhat limited due to the nature of the signals produced by the presence such faults. The characteristic frequencies that were found for the pulses created by different ball bearing faults cannot be found in the spectra of the raw signals recorded on a bearing $[1,3,7]$. A basic ball bearing fault detection procedure might apply enveloping and calculation of the cepstrum [7]. Another example for a simple ball bearing detection method might involve the calculation of the kurtosis of the time domain signal. Unfortunately such techniques would normally work for cases of a well developed fault and when there is not a lot of interference with other higher energy components coming from noise and/or other high frequency components.

The detection of real faults becomes problematic since the interest is primarily at detecting the faults at an early stage, when they are rather small. Then the pulses created by the fault will be of very low amplitude and will be impossible to detect in the raw signal or spectrum. A lot of the suggested fault detection methods for ball bearings involve a noise reduction in the recorded signals to facilitate the detection of the pulses created by the fault(s) $[2,3,5,6]$. A successful detection procedure should be able to first extract the appropriate frequency content of the measured signal and then detect the presence of faults in the extracted frequency band. Thus a lot of the ball bearing fault detection methods include a certain pre-processing and filtering to extract the proper frequency range from the bearing signature, clear the high energy content of the signal and prepare it for the detection process $[1,3,4,6,9]$. Different wavelet decompositions, filtering and enveloping are among the most frequently used 
techniques for the purpose $[1-4,6,7,10,11]$. The next stage after the preprocessing is designed to detect and eventually localize (qualify) the fault. The cepstrum and different variations of it is a popular signal processing technique for detecting ball bearing faults $[7,8]$. The spectral kurtosis is an alternative of the kurtosis which appears more promising and was successfully applied by different authors $[6,12,13]$. However after the application of all the procedures the decision whether a fault is present or not has to be taken by an expert through e.g introducing a threshold value of the considered feature or just on the basis of comparison to previous levels $[2,3,5,6,8,27,30]$. This paper suggests a method which does not require that: once the wavelet filtering is done and the principal components are extracted the PR procedure automatically issues a decision on whether a fault is present in the bearing or not. Thus the method suggested here has the potential to be made completely automatic /and computerized/ and hence very easy to apply without the intervention of experienced personnel.

The fault identification (localization) is for most methods the next stage which is done after the fault has been detected. On most occasions it is done utilizing the specific frequencies generated by different defects. These frequencies depend on the rotational speed, the number of balls and their geometry. The running speed is often unknown and/or unmeasurable. In addition, even if the type of the bearing in the machine is known, the manufacturer may not be able to guarantee the number of balls in a given bearing [28]. Thus on some occasions the precise determination of the specific fault frequencies might be difficult or even impossible which in turn might prevent the determination of the type of fault present. There are papers that specifically address the determination of the running speed and these specific fault frequencies [28,31]. Thus it is beneficial if a fault identification procedure does not 
rely on these specific frequencies. The procedure offered here does not use the specific frequencies generated by the three fault types considered. Moreover no expert intervention and analysis is required in order to recognize the fault present. It should be noted that most fault identification procedures do require a consequent analysis and the identification is normally done detecting the specific fault frequency

$[1-3,5,6,8,9,12-14,16,18]$. There are several methods that offer an automatic fault detection like the ones discussed in $[4,27,29]$ and most of them are based on pattern recognition. But they do not offer automatic identification of the fault type. To our knowledge the only method that offers an automated approach for fault detection is the one suggested in [27] and it deals with faults in a gearbox. The method presented in [11] has the potential and to our opinion could be made automatic, but the authors do not offer such an enhancement. The method suggested in this study offers an automatic detection and qualification (type identification) of faults in ball bearings without the need to estimate the specific fault frequencies. To our opinion these are very important features of the suggested method, because methods of such type have the potential of being easily incorporated and used for fault detection in real systems.

The simplicity of the procedure suggested should not be neglected as well. As it was mentioned the few methods that offer automatic fault detection are based on pattern recognition, but they tend to apply rather complex processes like neural nets and/or Markov models, neural-fuzzy inference and expert systems for the purpose [4,11]. The PR method used here is a rather simple and intuitive one and is based on the one nearest neighbour approach [21]. Hence it does not require large computational resources and its performance is normally very fast. Another feature that facilitates the recognition process to a great extent is the application of a modified PC method which is much more appropriate for categorical data [20,26]. The method 
is discussed in \&5. However it should be noted that most studies that feature application of PCA for fault detection apply the standard procedure and the use of the modified one is an enhancement which makes the fault recognition possible [23$25,27,32]$.

The rest of the paper is organized as follows. Paragraph \&2 deals with the manifestation of faults in rolling element bearings in the signals measured on the bearings and summarizes very briefly the suggested method. The next three paragraphs introduce the basic elements of the procedure suggested: $\mathbf{\&} 3$ introduces briefly the wavelet transform and its application for our purposes. \&4 formulates the problem considered as a PR problem and $\mathbf{8 5}$ presents the idea of PCA and the modified PCA used here as well as the way it is applied in this particular case. The experimental setup used is introduced in $\mathbf{\& 6}$. In $\mathbf{\&} 7$ some results, obtained using the suggested procedure, are presented. The paper concludes with a discussion of the presented methodology and the results obtained.

\section{\&2. Rolling element bearings and manifestation of faults in them.}

Rolling element bearings represent a class of bearings in which the main load is transferred through elements in rolling contact (rather than in sliding contact). The main components of such bearing are the outer race, the inner race, the cage and the rolling elements as shown in Fig. 1. In a rolling element bearing, impulses are created when a defect on a rolling surface impacts with another surface. Each time the defect comes in contact with another surface it makes the bearing vibrate at its natural frequency. This is why rolling element bearing defects are known as high frequency faults- in the first instance they can be detected in the frequency range where structural resonances dominate the spectrum. Unfortunately these are rather low 
energy pulses that can be easily buried in high energy low frequency signals and noise. The impulses are generated almost periodically and their frequency depends on the type (location) of defect, that is whether it is an inner race, an outer race or rolling element defect. There are several characteristic frequencies which determine the spacing of short pulses for each type of fault. It is this characteristic frequency which is of interest because it can be used to determine the type of fault- e.g. inner race fault (IRF), outer race fault (ORF) or rolling element fault (REF) [3,7]. So the information about the defect is contained in the spacing of the impulses rather than in their frequency content. However in practice, the spacing of pulses varies randomly to a certain extent due to varying load angle and slip as well as due to noise. Thus as a result of a number of random effects it becomes difficult to first find the fault in the high frequency range and secondly to determine its type.

This study suggests to consider the problem for detection and type identification of ball bearing faults as a pattern recognition (PR) problem. The signals are first subjected to a wavelet transform in order to extract the appropriate high frequency range and as a noise cleaning procedure. The obtained signals are then subjected to PCA. Only the first six principal components of the obtained signals are used as features for the recognition process. The following paragraphs explain briefly each of the procedures used and the motivation for using it as part of the process of signal analysis for ball bearing fault detection and identification.

\section{\&3. The wavelet transform}

The wavelet transform is a time frequency representation of a signal. It uses a window which is shifted along the signal and for every position the spectrum is calculated. Then this process is repeated many times with a different window for every new 
cycle. The result is a collection of time-frequency representations with different resolutions. The continuous wavelet transform of a signal $f(t)$ is given by:

$$
\gamma(s, \tau)=\int f(t) \Psi_{s, \tau}^{*}(t) d t
$$

Where * denotes the complex conjugate. The above equation shows how a signal $f(t)$ is decomposed into a set of wavelets $\Psi_{s, \tau}(t)$, where $s$ and $\tau$, scale and translation, are the new variables after the transform and $\gamma(s, \tau)$ are the wavelet coefficients. $\Psi(t)$ is the mother wavelet and $\Psi_{S, \tau}(t)$ are generated by scaling and translation:

$$
\Psi_{S, \tau}(t)=\frac{1}{\sqrt{s}} \Psi\left(\frac{t-\tau}{s}\right)
$$

The following gives how the signal $f(t)$ can be reconstructed using a wavelet basis:

$$
f(t)=\int_{-\infty}^{\infty} \int_{s>0}^{\infty} \gamma(s, \tau) \Psi_{s, \tau}(t) d s d \tau
$$

In the above equations (1)-(3) the wavelet basis functions are not specified. There are a number of wavelets that have been designed and they share the same (similar) general properties. The discrete wavelet transform is derived from the continuous wavelet

$$
\Psi_{j, k}(t)=\frac{1}{\sqrt{2^{j}}} \Psi\left(\frac{t-2^{j} k}{2^{j}}\right),
$$

where $s$ and $\tau$ are replaced by $2^{j}$ and $2^{j} k$. One of the fundamental properties of a wavelet is that it can decompose a signal by filtering it into a low frequency and a high frequency part. Thus if one is interested in the high frequency part (which is the case for ball bearing fault detection) one may keep the signal more or less intact and discard the low frequency part. By discarding the smooth low frequency part of the signal one can identify areas of impulsiveness, i.e. where the original signal changes 
quickly. And the areas of impulsiveness are exactly what we are looking for when we are interested in the short pulses crated by a ball bearing fault. This basic idea of decomposing a signal is represented in Figure 2. Figure 2 also represents the basic steps of such a decomposition by filtering as suggested in e.g. [19].

\section{Figure 2 somewhere here}

The above decomposition results in two coefficient vectors $\mathbf{c L}$ and $\mathbf{c H}$ which approximate the low frequency and the high frequency parts of the signal respectively. The $2 \downarrow$ block in Fig. 2 represents the down sampling where the odd coefficients of the filtered signal are omitted. Thus the number of coefficients produced by the above algorithm is approximately the same as the number of elements in the original discrete signal $f$. Applying the wavelet transform one compresses the signal and retains most of the information contained in its high frequency and its low frequency parts. The wavelet is a hierarchical transform and it can be applied on several levels $[4,19]$. Some authors suggest that for ball bearing fault detection $[4,6]$. In this study we only decompose the signal to the first level and take its high frequency part $\mathbf{c H}$. In this particular case applying the transform further to higher levels did not seem beneficial for our purposes.

\section{\&4. The problem considered as a PR problem.}

The problem considered in this paper is to detect a ball bearing fault and to identify its type i.e. recognize between different fault types. Three types of faults are considered in this investigation- outer race, inner race and rolling element faults. The problem is treated as a pattern recognition one. The categories (classes) at hand are related to 
each fault type including the no-fault category. Thus we want to recognize among four categories (classes)- no fault (NF), inner race fault (IRF), outer race fault (ORF) and rolling element fault (REF). This can be done using the measured acceleration signals on the bearing. In this case each signal is first subjected to wavelet filtering and the vector $\mathbf{c H}$ representing its detailed (high frequency) coefficients is used to approximate the original signal $f$.

So the task is to distinguish between the four categories (classes) of vectors $\mathbf{c H}$. A PR problem normally has two stages, a feature extraction stage and the actual recognition stage. The feature extraction stage extracts some parameters from the vectors $\mathbf{~} \mathbf{H}$ which form the so called pattern vectors that are further used to distinguish between the defined categories. Thus each signal corresponding to a certain state from the above categories will be characterized by its unique pattern vector c. Principal component analysis (PCA) [20] is the method used here to extract relevant features and form the pattern vectors. The first $k$ principal components $\mathbf{c}, \mathbf{c}=\left[c_{1}, c_{2}, \ldots, c_{k}\right]^{\mathrm{T}}$ are then used as features (in our case $k=6$ ). The next paragraph describes the idea of PCA, the modification used here and how the method is applied for this particular case.

In this study the actual recognition is done using the 1-nearest neighbour ( 1$\mathrm{NN})$ method. The 1-NN method classifies a vector $\mathbf{c}$ to the category to which its nearest neighbour belongs. The nearest neighbour is found using the Euclidean distance between two pattern vectors:

$$
D_{i}=\left\|\mathbf{c}-\mathbf{c}_{\mathbf{i}}\right\|=\sqrt{\left(\mathbf{c}-\mathbf{c}_{\mathbf{i}}\right)^{T}\left(\mathbf{c}-\mathbf{c}_{\mathbf{i}}\right)}
$$

The 1-NN classifier finds the nearest neighbor of $\mathbf{c}, \mathbf{c}_{\mathbf{i}}$, and assigns $\mathbf{c}$ to the class of $\mathbf{c}_{\mathbf{i}}$. In order to apply this classifier one needs a 'training' sample of vectors $\mathbf{c}_{\mathbf{j}}$, among 
which the classifier will look for the NN. The construction of the training sample is discussed in $\& 6$.

\section{\&5. Principal component analysis (PCA).}

The main idea of PCA is to reduce the dimensionality of a signal in the time or in the frequency domain while retaining most of the variance present in the data. PCA transforms the signals $\mathbf{c H}$, into new pattern vectors $\mathbf{c}$ whose components are called principal components (PCs). The new PCs are uncorrelated. In general the first several PCs are responsible for a considerable part of the variance contained in the signals. PCA is done using the autocorrelation matrix of the vectors $\mathbf{c H}$. It is suggested here to use a modified PCA. This modified method has some advantages when applied to categorical data which will be discussed later. It uses the generalised autocorrelation matrix, which is calculated for the whole set of measurements, rather than the autocorrelation matrices corresponding to each category $[20,25,26]$. It is determined using the following relation:

$$
\mathbf{R}=\sum_{i=1}^{M} p\left(\omega_{i}\right) E\left(\overline{\mathbf{C}}_{\mathbf{H}_{\mathbf{i}}} \cdot \overline{\mathbf{C}}_{\mathbf{H}_{\mathbf{i}}}^{T}\right)
$$

where $M$ is the number of categories (M=4 in this case), $p\left(\omega_{i}\right)$ is the a priori probability for the category $\omega_{i}$, the superscript ' $\mathrm{T}$ ' stands for transpose, $E$ denotes the mathematical expectation, and $\overline{\mathbf{C}} \overline{\mathbf{H}}_{\mathbf{i}}$ is a standardised vector from the $i$-th category. In the absence of any other information the a priori probabilities for the categories $\omega_{i}$ are assumed equal $p\left(\omega_{i}\right)=1 / M$. The first $k$ largest eigenvalues of the correlation matrix $\mathbf{R}$ and their associated eigenvectors $\Phi_{i}$ are found. The vectors $\Phi_{i}$ are packed to form the transformation matrix $\Phi$ 


$$
\boldsymbol{\Phi}=\left[\Phi_{1}, \Phi_{2}, \ldots, \Phi_{k}\right]
$$

A vector $\mathbf{C H}$ can be then transformed into a new feature $\mathbf{c}$ vector via

$$
\mathbf{c}=\boldsymbol{\Phi}^{T} \cdot \mathbf{C H}
$$

where $\boldsymbol{\Phi}^{T}$. is the transpose of $\boldsymbol{\Phi}$.

The primary idea of PCA is to transform an original vector $\mathbf{C H}$ into a new vector $\mathbf{c}$, which has a smaller dimension $k, k<<p$. The choice of the $k$ new variables is based on the variance that they are responsible for. When applied to categorical data the modified PCA used here (equation (6)) is expected to have other advantageous properties since it decreases the inter-class variance while at the same time increasing the between-class variance. This results in 'clustering' the new PC vectors $\mathbf{c}_{\mathrm{j}}$ or bringing together the pattern vectors from the same category while at the same time increasing the distance between pattern vectors from different categories $[21,25,26]$. In this particular case PCA is aimed to first extract most of the 'detailed' information contained in the vectors $\mathbf{C H}$, since it retains most of their variability, and then group the obtained pattern vectors $\mathbf{c}$ into the defined four categories NF,IRF,ORF and REF.

PCA has been used for a number of application including signal analysis and especially dynamic (acceleration) signal analysis [23-26]. In its dynamics applications it is indeed most frequently used for vibration-based damage and fault detection [2224,26]. A number of articles suggest its application for structural damage detection $[23,24,26]$. The case of structural damage and its detection from vibration measurements is somewhat similar to the detection of ball bearing faults. The presence of a fault can be seen to change some frequency ranges and the lines in these 
frequency ranges are further used as variables, which are subjected to PCA [24,26]. In the present study a similar approach is taken by first applying the wavelet transform, which actually selects the frequency range containing the variability due to the presence of a fault, and then subjecting the already selected frequency lines to PCA to choose and weight properly only those, which are responsible for the biggest part of the between-class variance.

\section{\&6. The experiment, the signals and how the method works.}

The experimental setup detailed in Figures 3a and 3b was used to record signals corresponding to the different conditions. The power is provided by an electrical motor.

\section{Figures $3 \mathrm{a}$ and $3 \mathrm{~b}$ somewhere here}

To measure the vibrations an accelerometer is mounted on the square housing of the analysed bearing. The bearings analysed are single row deep groove ball bearings with eight balls (SKF 6308). Four different bearings were used to study the four categories namely one bearing with no fault (NF), one with an inner race fault (IRF), one with an outer race fault (ORF) and one with a rolling element fault (REF). The faults considered here are very small notches. Thus the primary interest of this study is to detect and identify very small faults at a rather early stage. Bigger faults are not considered since they are much easier to detect and identify. A bearing with an inner race fault is shown in Figure 4. 
Figures 5 and 6 represents raw signals from the four categories and the spectra from the three fault categories, respectively. The specific fault frequencies are circled on Fig 6. It can be appreciated that the signals are different but it is impossible to recognise the differences and thus distinguish between the four categories. The frequency domain signals also cannot be used to give any clear indication for the signal type. So it is obvious that further transformation and analysis of the raw signals is necessary.

\section{Figure 5 and Fig 6 somewhere here}

Each of the signals is then subjected to a wavelet transform using Daubechies2 (db2) and its high frequency part represented by the vector $\mathbf{C H}$ is taken. Figure 7 gives the details of the bandwidths of the low and high frequency filters.

\section{Figure 7 somewhere here}

The impulses created by bearing defects are of high frequency nature and the high frequency part $\mathbf{C H}$ of the decomposed signal is considered further. Figure $\mathbf{8}$ presents signals coming from the three different types of faulty bearings (IRF, ORF, REF), after the wavelet transform. The periods corresponding to each specific type of fault are indicated by arrows on each plot. The visual observations of these plots shows that the impacts are visible but it is still difficult to determine the intervals 
between impacts which makes difficult the identification of the bearing condition/category vs. NF, IRF,ORF or REF.

\section{Figure 8 somewhere here}

The next step is the application of the PCA to reduce the number of coefficients $\mathbf{C H}$ and extract relevant features. In this case we used the first six principal components, which were responsible for more than $90 \%$ of the whole variance.

400 signals (100 from each category) were measured and used for training purposes. The training signals are used to estimate the autocorrelation matrix $\mathrm{R}$ (equation (6) ) and to find a sample of pattern vectors $\mathbf{c}_{i j}, i=1, \ldots, 100$, with known categories $j, j=1,2,3,4,1-N F, 2-I R F, 3-O R F, 4-R E F$. The a priori probabilities for the four categories were assumed equal, $p\left(\omega_{i}\right)=0.25$. For illustration purposes the first two principal components of some signals from the four different categories are shown in Figure 9. The clustering of the signals according to the introduced categories is already visible. The first 6 PC's are used to form the sample of pattern vectors $\mathbf{c}_{\mathrm{ij}}$ which will be used for recognition purposes.

\section{Figure 9 somewhere here}

Another 400 signals (100 from each category) were measured and used for testing purposes. They are also subjected to PCA with the already obtained matrix $\mathbf{R}$ 
and their first 6 principal components are taken to form their pattern vectors. Then the $1-\mathrm{NN}$ method ( see \&4) is applied to identify the category of each of these vectors.

The whole procedure used to detect and recognise faults in ball bearings is depicted in Figure 10. The identification results with the pattern vectors from the testing sample are shown in Table 1 . The results show very good recognition rate despite that the initial vectors $\mathbf{C H}$ were not recognisable using visual inspection (see Figure 8).

\section{Figure 10 somewhere here}

\section{Table 1 somewhere here}

We would like to briefly mention that the procedure developed was also tested to recognise bigger faults. The faults were extended and the new signals were offered for recognition to the already developed system. It is worth mentioning that these faults were readily recognised with even higher success rate than the small faults. But since the primary interest here (and in general) is in detecting and recognising small faults and bigger faults are much easier to detect and identify, these results are not given and they are not discussed any further.

\section{\&7. Discussion and some deductions.}

This study presents a method, which offers an automatic detection and identification of ball bearing faults. The method is developed and tested for very small faults which is the primary interest of such procedures when the faults are very 
difficult to detect. It is noted that unlike most procedures offered for ball bearing fault detection the method suggested does not concentrate on making the fault impulses visible, it attempts to make the signals coming from different bearing conditions more distinguishable. This is achieved by applying a modified PCA approach which is known to enhance the separability of categorical data. The PCA is applied on the already filtered high frequency content signals which contain the information about the presence of faults. It takes those components of the $\mathbf{C H}$ vectors, which are responsible for most of the variability between the different categories and gives the highest weights to the components with the highest variability (the components that actually make the categories different). As can be seen from the results presented in Figure 9, even if only the first two PCs are used the effect of clustering is obvious and the four introduced signal/bearing categories are already visible. A simple PR procedure based on the 1 nearest neighbour approach is applied to achieve the fault detection and identification. It shows very high separation rate, namely between $94 \%$ and $96 \%$ of the faults are correctly detected and recognised. It should be noted that these results refer to the signals from the testing sample only. Most studies on ball bearing fault detection do not offer such results, since they tend to concentrate on the amplification of the small pulses created by a fault and on improving their visibility and detectability. Where similar results are offered the success rate seems to be somewhat lower. In [4] for instance similar results are offered which give the success rate of different methods in recognising single and multiple faults in ball bearings. It should be noted that some of the methods give a success rate lower than $50 \%$. Only the last method which is the most complicated one gives a rather high success rate of $99 \%$. 
The suggested method is automatic which makes it very appropriate for potential practical applications since unlike most other methods it does not require any human and/or expert intervention to perform the detection and the identification process. This quality combined with the high success rate of the method gives us confidence that it can be developed and successfully used for industrial applications.

In conclusion it should be noted that in this paper the method is demonstrated for a specific type of setup/machine and in order to apply it to another machine or if the measurement point is changed a different training sample should be created. The procedure is still the same so it just a matter of making another training sample to estimate the new autocorrelation matrix. It is worth pointing out that this is the case with most methods that use e.g neural nets and/or pattern recognition for fault detection in machinery and /or structures [4,26,27,29]. The method is usually demonstrated for a specific machine/ setup but the application to another machine/ structure requires certain modifications like generating a new training sample. 


\section{References}

1. X. Lou, K. A. Loparo, Bearing fault diagnosis based on wavelet transform and fuzzy inference, Mechanical Systems and Signal Processing 18 (2004) 10771095.

2. S. Bozchalooi, M. Liang, A joint resonance frequency estimation and inband noise reduction method for enhancing the detectability of bearing fault signals, Mechanical Systems and Signal Processing 22 (2008) 915-933.

3. N. Sawalhi, R.B. Randall, H. Endo, The enhancement of fault detection and diagnosis in rolling element bearings using minimum entropy deconvolution combined with spectral kurtosis, Mechanical Systems and Signal Processing 21 (2007) 2616-2633.

4. V. Purushotham, S. Narayanan, S.A.N. Prasad, Multi-fault diagnosis of rolling bearing elements using wavelet analysis and hidden Markov model based fault recognition, NDT\&E International 38 (2005) 654-664.

5. J.P. Dron, L. Rasolofondraibe, F. Bolaers, A. Pavan, High-resolution methods in vibratory analysis: application to ball bearing monitoring and production machine, International Journal of Solids and Structures 38 (2001), 4293-4313

6. S. Prabhakar, A.R. Mohanty, A.S Sekhar, Application of discrete wavelet transform for detection of ball bearing race faults, Tribology International 35 (2002) 793-800.

7. S. A. McInerny and Y. Dai, Basic Vibration Signal Processing for Bearing Fault Detection, IEEE Transactions on Education, 46 (1) (2003) 149-156. 
8. Choi Young-Chul and Kim Yang-Hann, Fault detection in a ball bearing system using minimum variance cepstrum, Meas. Sci. Technol. 18 (2007) 14331440.

9. J. P. Dron, Fault detection and monitoring of a ball bearing benchtest and a production machine via autoregressive spectrum analysis, Journal of Sound and Vibration 218(3) (1998) 501-5125

10. C. Smith, C. M. Akujuobi, P. Hamory, K. Kloesel, An approach to vibration analysis using wavelets in an application of aircraft health monitoring, Mechanical Systems and Signal Processing 21 (2007) 1255-1272

11. Q. Sun, Y. Tang, Singularity analysis using continuous wavelet transform for bearing fault diagnosis, Mechanical Systems and Signal Processing (2002) 16(6) 1025-1041.

12. N. Sawalhi, R. Randall, The application of spectral kurtosis to bearing diagnosis, Acoustic Conference, Gold Coast, Australia, Nov. 2004

13. R.B. Randall, J. Antoni, N. Sawalhi, Application of spectral kurtosis to bearing fault detection in rolling element bearings, 11-th International Congress Sound and Vibration(ICSV11) St Petersburg, 2004.

14. J. Lin, L. Qu, Feature extraction based on Morlet wavelet and its application in mechanical fault diagnosis, Journal of Sound and Vibration 234 (2000) 135-148.

15. J. Lin, M.J. Zuo, K.R. Fyfe, Mechanical fault detection based on the wavelet de-noising technique, Journal of Vibration and Acoustics 126 (2004) 9-1

16. Kim Y-H, Lim B-D and Cheoung W-S 1991 Fault detection in a ball bearing system using a moving window. Mech. Syst. Signal Process. 5 461-73 
17. Staszewski W J and Tomlinson G R 1997 Local tooth fault detection in gearboxes using moving window procedure, Mech. Syst. Signal Process. 11 33150.

18. H. Qiu, J. Lee, J. Lin, G. Yu, Wavelet filter-based weak signature detection method and its application on rolling element bearing prognosis, Journal of Sound and Vibration 289 (2006) 1066-1090.

19. S.G. Mallat, A theory for multiresolution signal decomposition: the wavelet representation, IEEE Trans Pattern Anal Machine Intelligence,11(7) (1989) 674-93.

20. I. Joliffe, Principal component analysis, 2nd edition, Springer series in statistics, Springer New York, USA 2004.

21. C. M. Bishop, Neural Networks for Pattern Recognition, Oxford University Press, Oxford, UK 1995.

22. N. Baydar, Q. Chen, A. Ball, U. Kruger, Detection Of Incipient Tooth Defect In Helical Gears Using Multivariate Statistics, Mechanical Systems and Signal Processing 15(2) (2001) 303-321.

23. L. Moniz, J.M Nichols, C.J. Nichols, M. Seaver, S.T. Trickey, M.D. Todd, L.M. Pecora, L.N. Virgin, A multivariate, attractor-based approach to structural health monitoring, Journal of Sound and Vibration 283 (2005) 295-310.

24. J.M. Nichols, S.T. Trickey, M. Seaver, Damage detection using multivariate recurrence quantification analysis, Mechanical Systems and Signal Processing 20 (2006) 421-437.

25. S.W. Choi, I.B. Lee, Nonlinear dynamic process monitoring based on dynamic kernel PCA, Chemical Engineering Science 59 (2004) 5897 - 5908. 
26. I. Trendafilova, M. Cartmell, W. Ostachowicz, Vibration based damage detection in an aircraft wing scaled model using principal component analysis and pattern recognition. Journal of Sound and Vibration 313 (3-5) 560-566

27. Saravanan N., V.N.S Kumar, Siddabattuni,K.I. Ramachandran, A comparative study on classification of features by SVM and PSVM extracted using Morlet wavelet for fault diagnosis of spur bevel gear box, Expert systems with applications, 35 (3), pp. 1351-1366.

28. Ocak H., Loparo K.A, Estimation of the running speed and bearing defect frequencies of an induction motor from vibration data, 2004 Mechanical Systems and Signal Processing18 (3), pp. 515-533.

29. David J. Van Dyke \& William A. Watts, Automated Rolling Contact Bearing Fault Detection Using Cepstrum Analysis, International Machinery Monitoring \& Diagnostic Conference, Las Vegas, 1989.

30. J.P.Dron, Rasolofondraibe, C.Couet and A.Pavan, Fault detection and monitoring of a ball bearing benchtest and a production machine via autoregressive spectrum analysis, Journal of Sound and Vibration 218 (3), pp. $501-525$

31. Wang, Y.-F., Kootsookos, P.J., Modelling of low shaft speed bearing faults for condition monitoring, Mechanical Systems and Signal Processing 12 (3), pp. 415-426

32. D.-M. Yang, Induction Motor Bearing Fault Detection with Non-stationary Signal Analysis, Proceedings of International Conference on Mechatronics Kumamoto Japan, May 2007. 


\section{Figure captions}

Figure 1. Main elements of a rolling element bearing and load distribution

Figure 2. Basic steps of wavelet signal decomposition

Figure 3a. Experimental setup.

Figure 3b. Sketch of the installation used

Figure 4. Bearing with an inner race fault

Figure 5. Raw signals from different categories

Figure 6. Wavelet decomposition with frequency bands used

Figure 7 Zoomed signals from the three fault categories after wavelet, with the corresponding periods shown

Figure 8. The first two PC's of some of the training vectors for the four different categories.

Figure 9. Schematic of the whole process used for fault recognition

Table 1. Percentage of correctly classified and miss-classified signals (faults) 


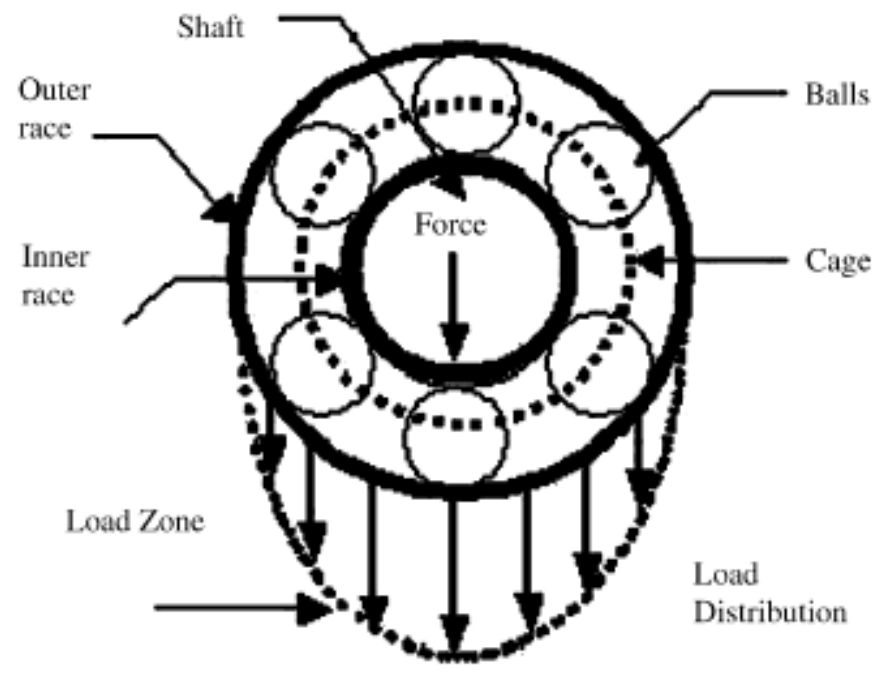

Figure 1. Main elements of a rolling element bearing and load distribution 


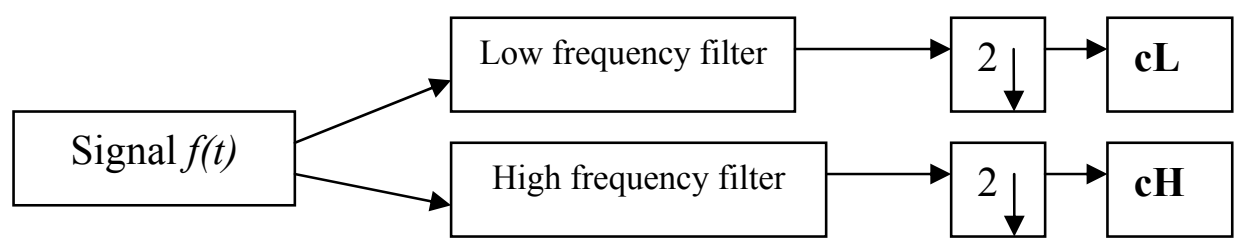

Figure 2._Basic steps of wavelet signal decomposition 


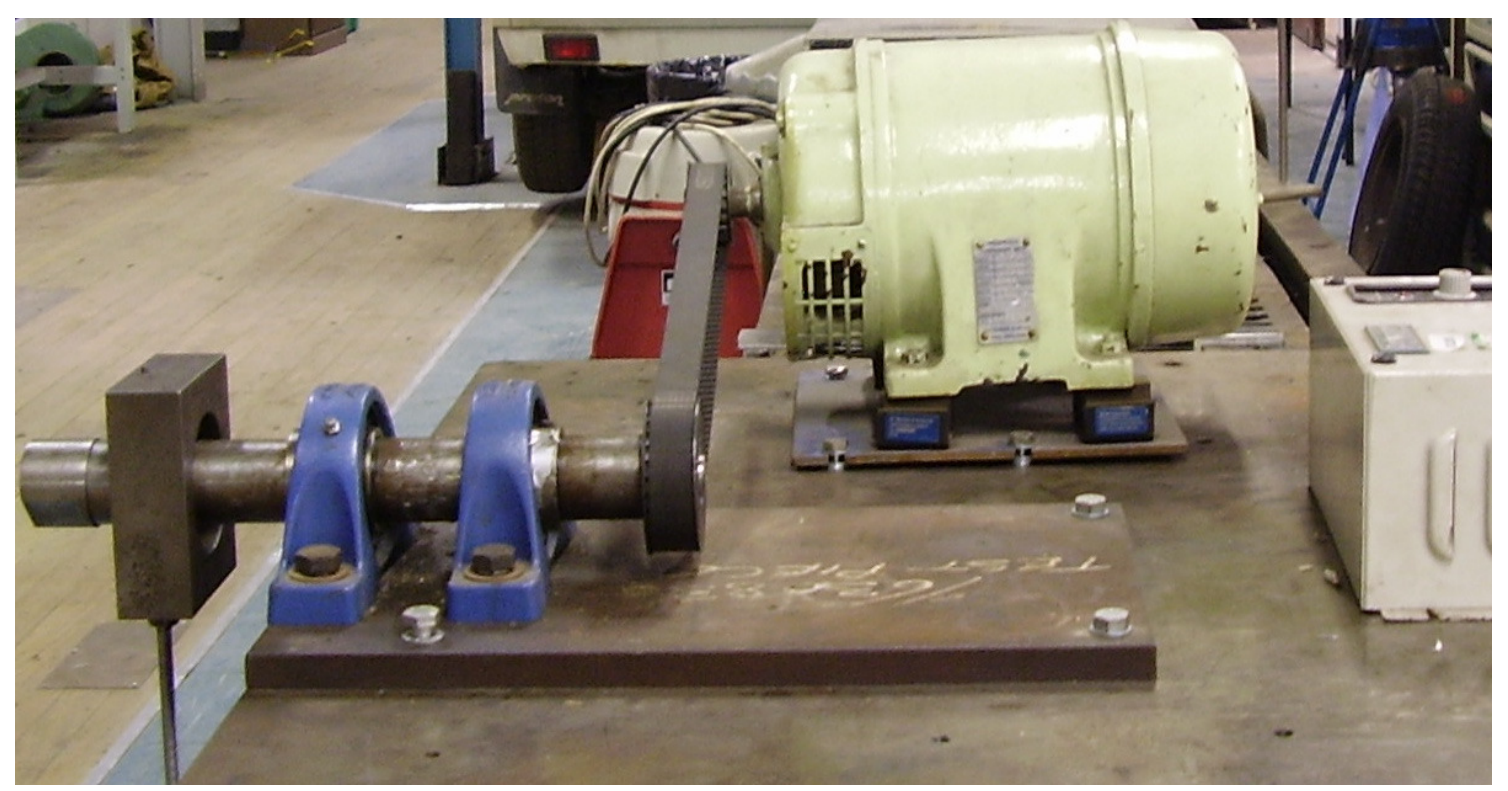

Figure 3a. Experimental setup. 

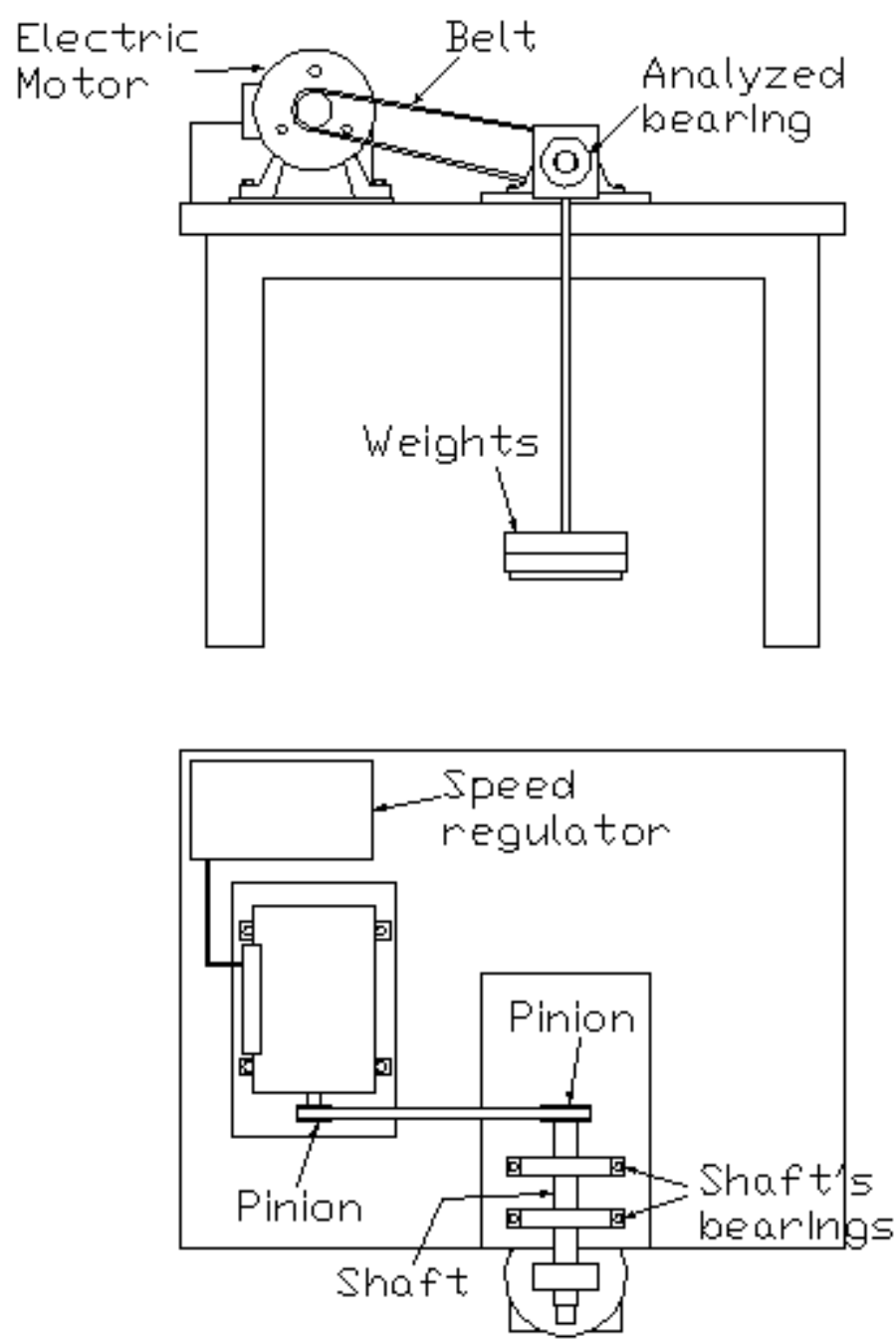

Figure 3b. Sketch of the installation used 


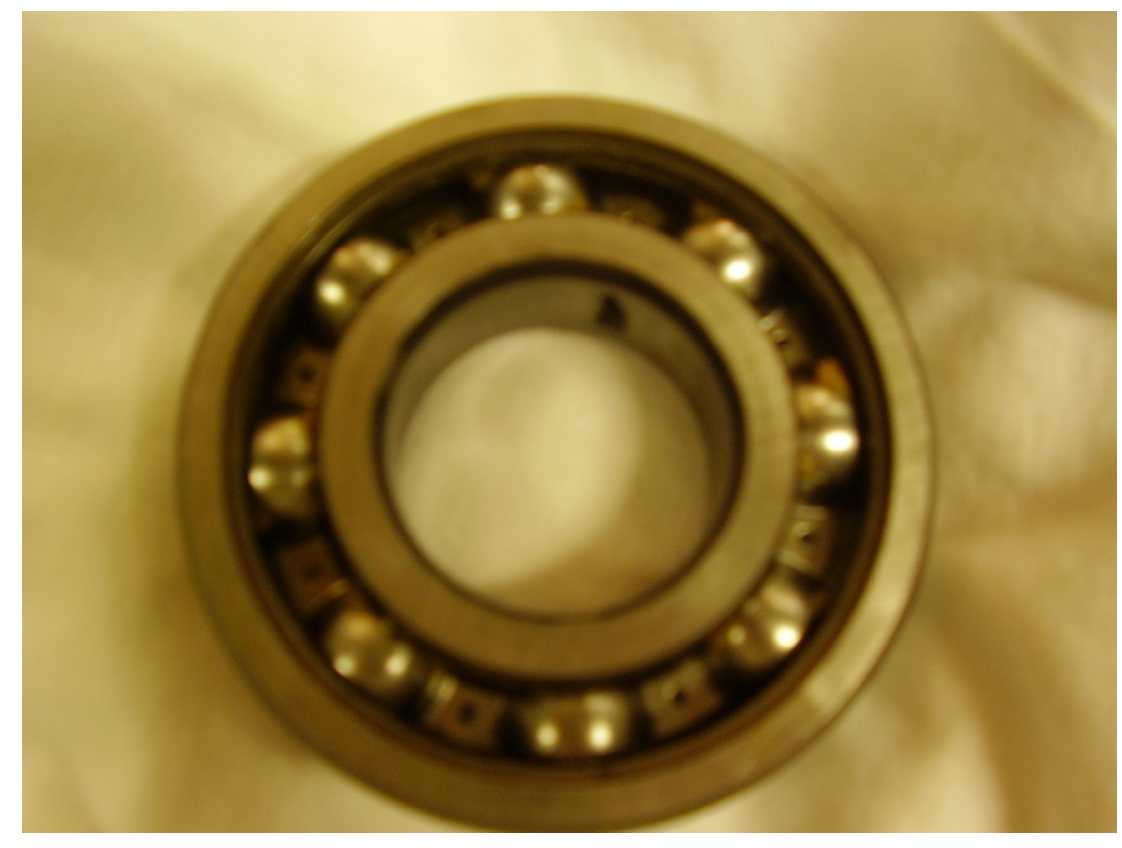

Figure 4. Bearing with an inner race fault 

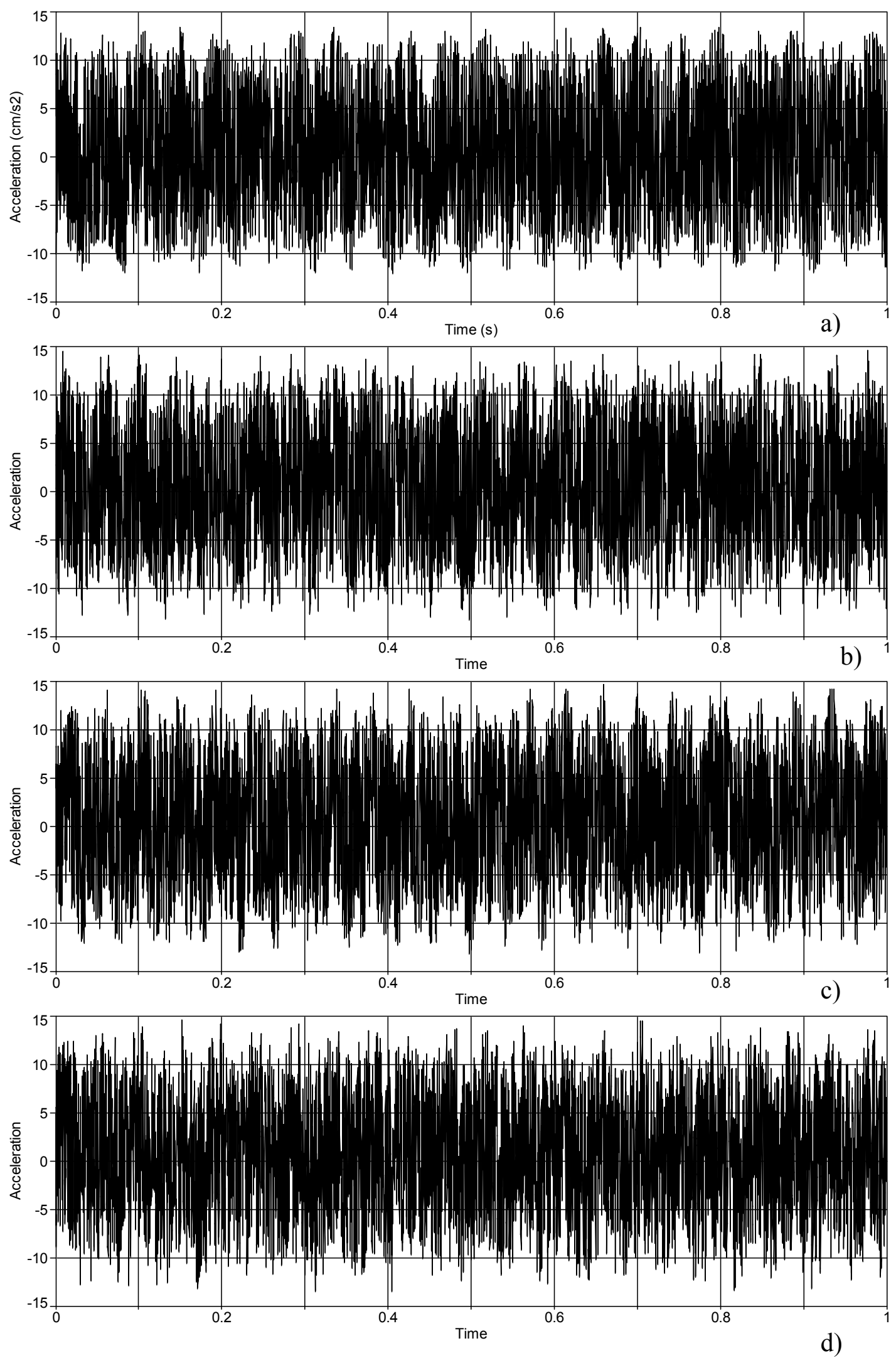

Figure 5. Raw signals from different categories a) no fault, b) inner race fault, c) outer race fault, d) rolling element fault 

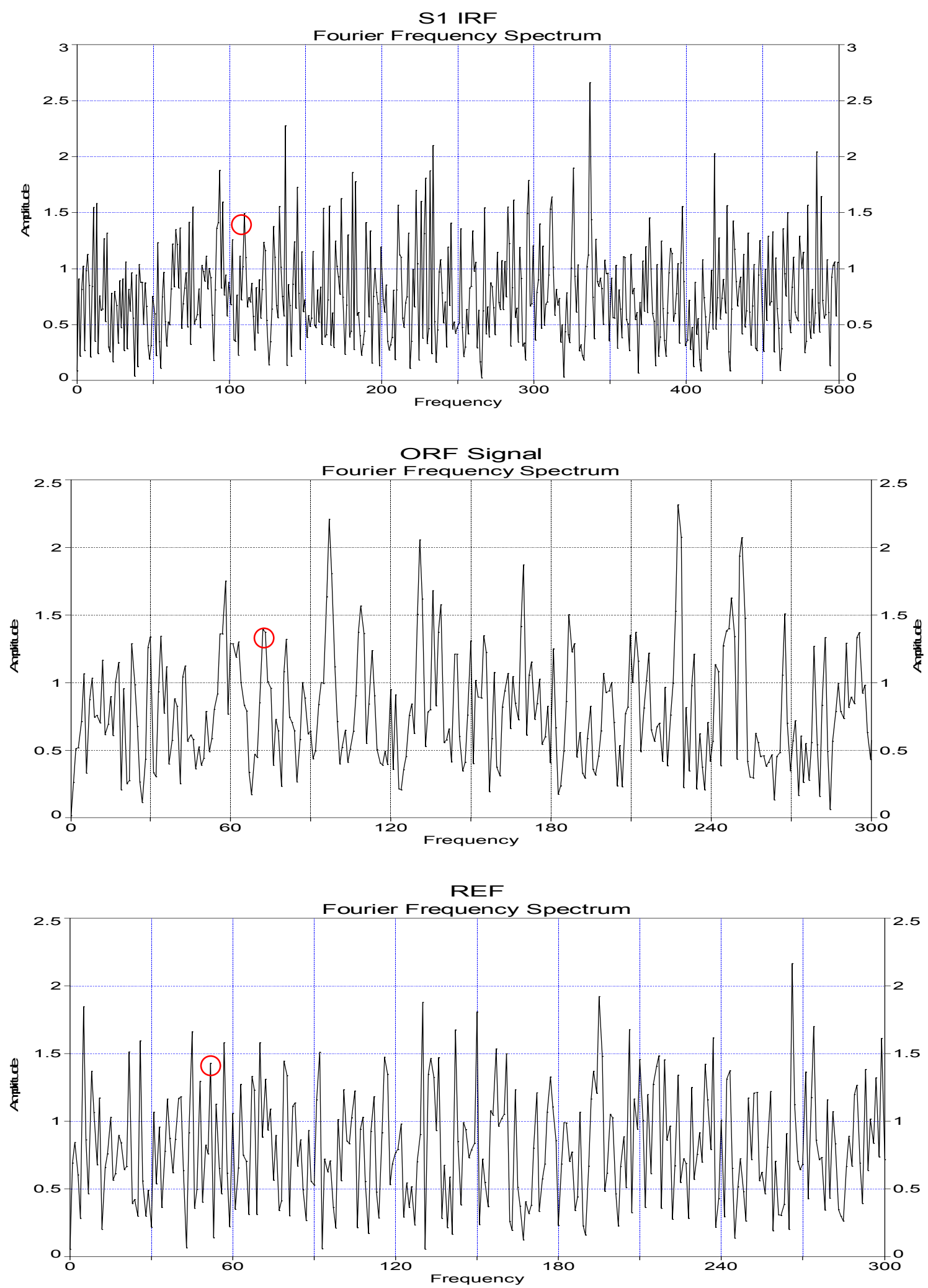

Figure 6. Spectra of the raw signals 


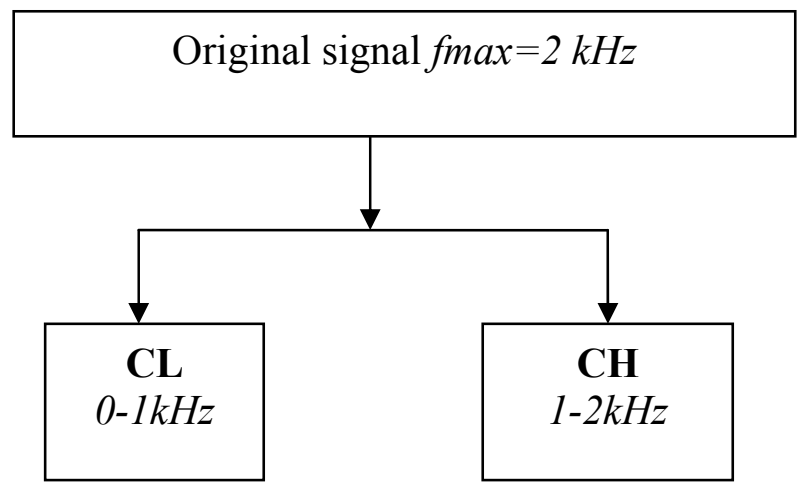

Figure 7. Wavelet decomposition with frequency bands used 

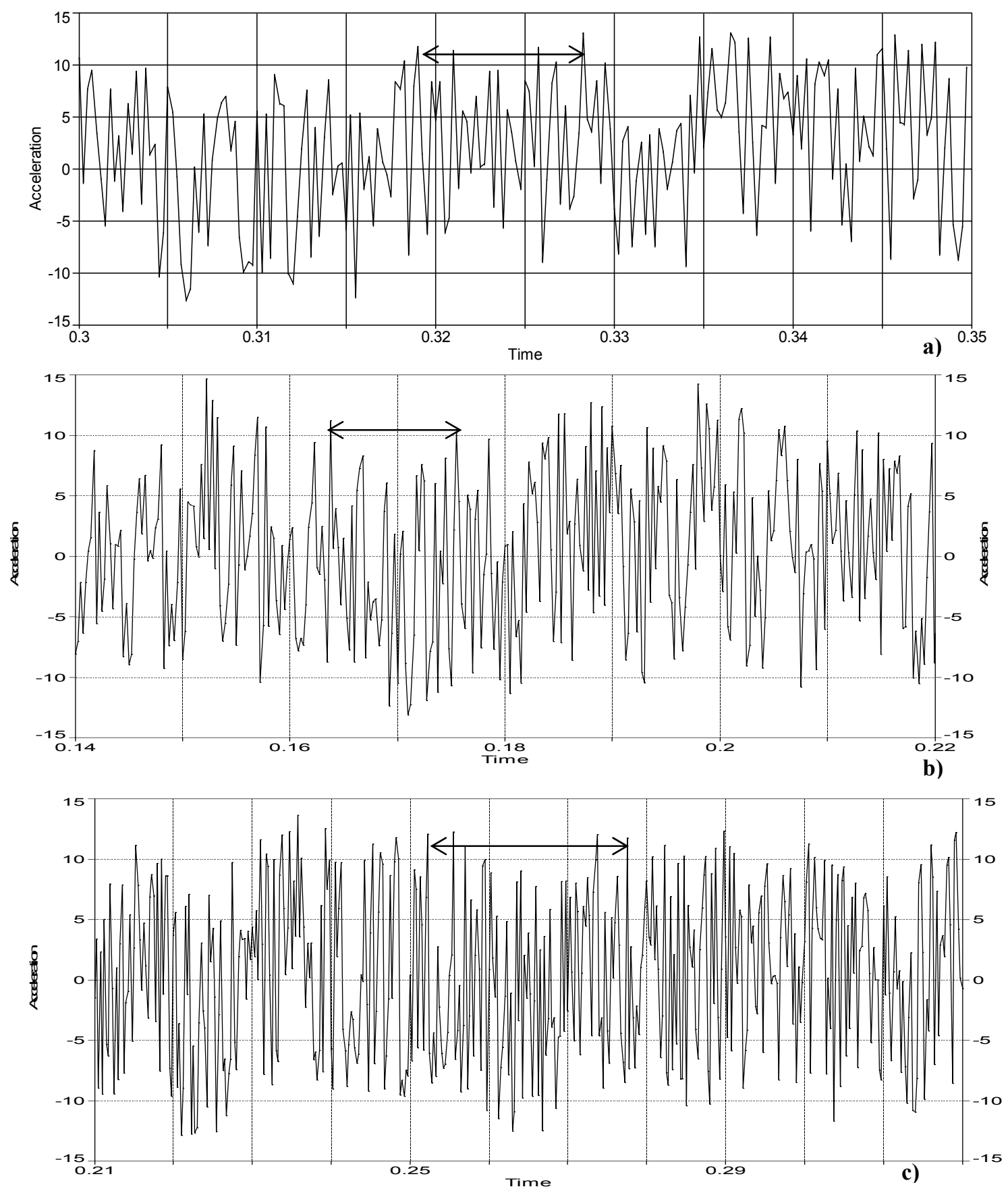

Figure 8 Zoomed signals from the three fault categories after wavelet, the corresponding periods are shown a) Inner race fault, b) Outer race fault, c) rolling element fault 


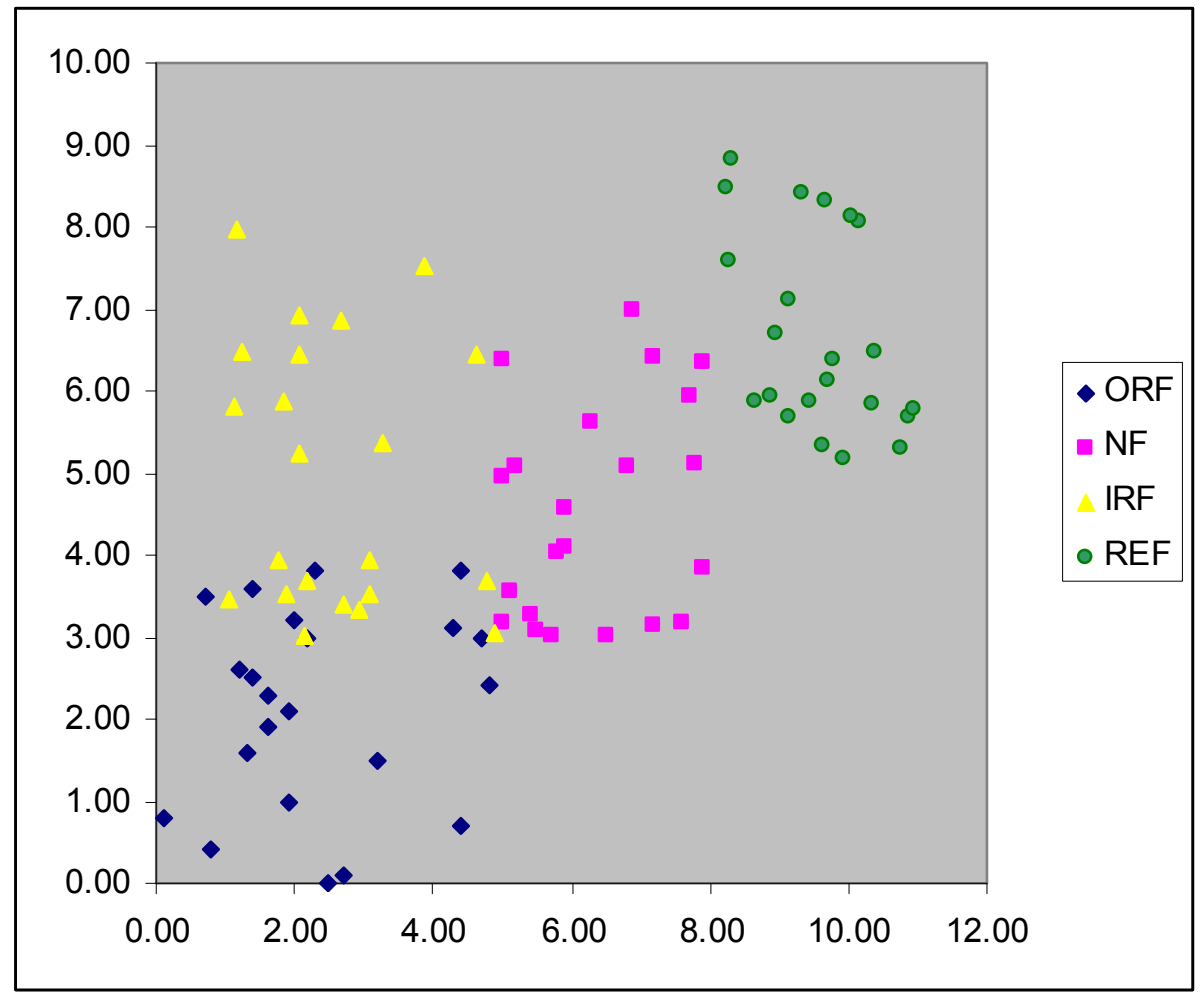

Figure 9. The first two PC's of some of the training vectors for the four different categories. The clustering effect is visible. 
Pattern recognition

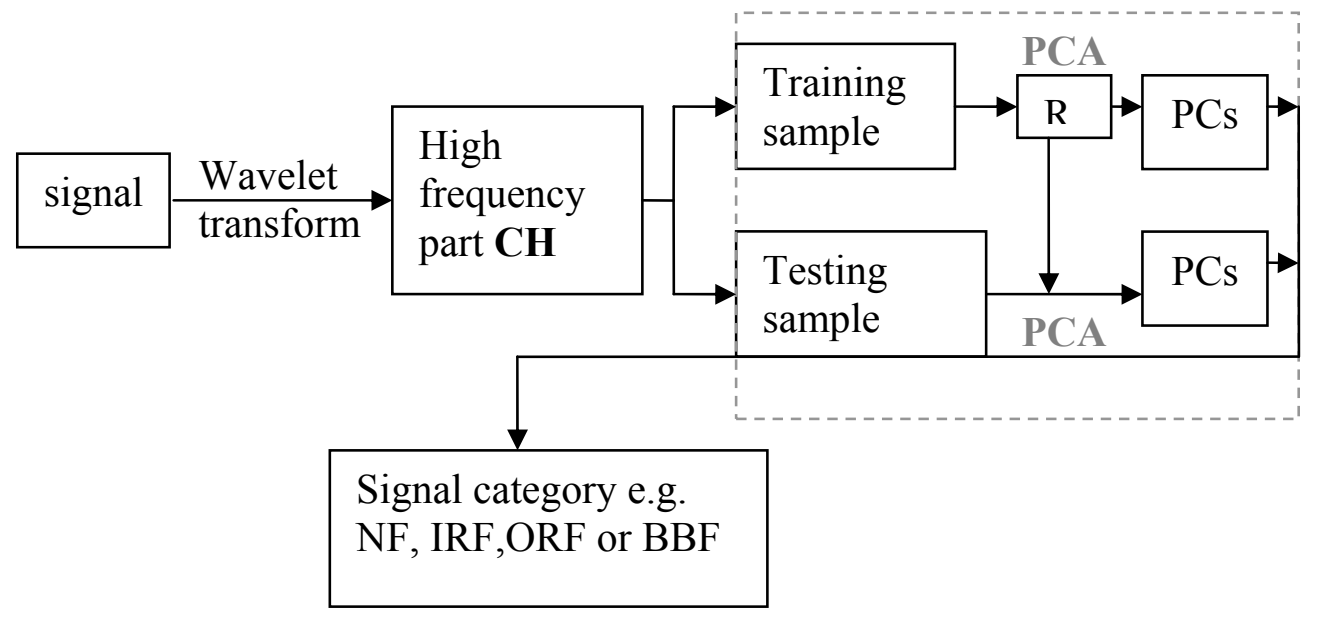

Figure 10. Schematic of the whole process used for fault recognition 


\begin{tabular}{|c|c|c|c|c|}
\hline $\begin{array}{c}\text { True } \\
\text { category }\end{array}$ & \multicolumn{5}{|c|}{ Recognised category } \\
\hline & NF & IRF & ORF & REF \\
\hline NF & $\mathbf{9 5}$ & 2 & 2 & 1 \\
\hline IRF & 1 & $\mathbf{9 6}$ & 3 & \\
\hline ORF & & 5 & 95 & $\mathbf{9 4}$ \\
\hline BF & 2 & 3 & 1 & \\
\hline
\end{tabular}

Table 1. Percentage of correctly classified and miss-classified signals (faults) 